\title{
DIFFERENTIABILITY OF SAMPLE FUNCTIONS IN GAUSSIAN PROCESSES ${ }^{1}$
}

\section{J. YEH}

1. Let $\{\xi(t), t \in T\}, T=[0,1]$, be a Gaussian process, defined on an underlying probability space $(X, \mathfrak{F}, P)$, in which $X$ is the collection of all real valued functions $x(t)$ defined on $T, \mathfrak{F}$ is the $\sigma$-field of subsets of $X$ generated by the Borel cylinders in $X$, and $\xi(t)$ is defined by

$$
\xi(t, x)=x(t) \text { for } t \in T, x \in X .
$$

Here, by a Borel cylinder in $X$, we mean a subset $B_{X}$ of $X$ defined by

$$
B_{X}=\left\{x \in X ;\left[x\left(t_{1}\right), \cdots, x\left(t_{n}\right)\right] \in B\right\}
$$

where $t_{1}, \cdots, t_{n} \in T$, and $B$ is a Borel set in the $n$-dimensional Euclidean space. Such a process exists according to the Kolmogoroff Extension Theorem. If, further,

$$
E\{\xi(t)\}=0 \text { for } t \in T,
$$

and for some $\beta, b>0$,

$$
E\left\{\left|\xi\left(t^{\prime}\right)-\xi\left(t^{\prime \prime}\right)\right|^{2}\right\} \leqq b\left|t^{\prime}-t^{\prime \prime}\right|^{\beta} \quad \text { for } t^{\prime}, t^{\prime \prime} \in T
$$

and the process is separable then ${ }^{2}$

$$
P^{*}\left(C_{\lambda}\right)=1 \text { for } 0<\lambda<\beta / 2
$$

where $P^{*}$ is the outer measure of $P$ and $C_{\lambda}$ is the subset of $X$ consisting of the Lipschitz $\lambda$-continuous elements, i.e., those functions which satisfy

$$
\left|x\left(t^{\prime}\right)-x\left(t^{\prime \prime}\right)\right| \leqq h\left|t^{\prime}-t^{\prime \prime}\right|^{\lambda} \quad \text { for } t^{\prime}, t^{\prime \prime} \in T
$$

with $h$ depending on $x$.

In this article we consider the differentiability of the sample paths $x(t)$ of the process $\{\xi(t), t \in T\}$. We shall assume that our process satisfies the additional condition that for some $\alpha, a>0$,

$$
E\left\{\left|\xi\left(t^{\prime}\right)-\xi\left(t^{\prime \prime}\right)\right|^{2}\right\} \geqq a\left|t^{\prime}-t^{\prime \prime}\right| \alpha \text { for } t^{\prime}, t^{\prime \prime} \in T .
$$

Our result is stated in the theorem in $\$ 2$, after a brief discussion of

Received by the editors June 10, 1966.

1 This research was supported in part by the National Science Foundation Grant GP 5436.

2 A proof for this statement can be found for instance in [4]. 
the measure induced on the space of continuous functions by the process. Our theorem implies in particular that, if $\alpha / 2<1$, then almost every sample function $x(t)$ is almost nowhere differentiable with respect to $t$ on $T$. This is an extension of the classical result of Wiener for the Brownian motion process.

2. Let $C$ be the subset of $X$ consisting of the continuous functions. Now that $C_{\lambda} \subset C$ and $P^{*}(C)=1$, the probability measure $P$ induces a measure $m_{G}$ on $C$ according to a theorem by Doob (Theorem 1.1, [1]). Specifically this measure is obtained as follows. Let $\mathfrak{F}_{c}$ be the field of Borel cylinders in $C$. A Borel cylinder $B_{C}$ in $C$ is a subset of $C$ of the type

$$
B_{C}=\left\{x \in C ;\left[x\left(t_{1}\right), \cdots, x\left(t_{n}\right)\right] \in B\right\}=B_{X} \cap C
$$

where $t_{1}, \cdots, t_{n} \in T, B$ is a Borel set in the $n$-dimensional Euclidean space and $B_{X}$ is as defined previously. If we now define a set function $m_{G}$ on $\mathfrak{F}_{C}$ by

$$
m_{G}\left(B_{C}\right)=P\left(B_{X}\right),
$$

then $m_{G}$ is well-defined according to the above quoted theorem by Doob and is in fact a measure on the field $\mathfrak{F}_{c}$. Finally by means of a Carathéodory extension we have a measure space $\left(C, \mathcal{S}^{*}, m_{G}\right)$ in which $\mathbb{S}^{*}$ is the $\sigma$-field of the Carathéodory measurable sets. Our theorem can now be stated.

Theorem. Let $\{\xi(t), t \in T\}, T=[0,1]$, be a separable Gaussian process satisfying the conditions (1), (2), and (3). Let $m_{G}$ be the measure induced on the space $C$ of continuous functions $x(t)$ defined on $T$. Let $\lambda>\alpha / 2$. Then for almost every $x \in C$ relative to $m_{G}$

$$
\begin{aligned}
& \lim _{s \downarrow 0} \sup \frac{|x(t+s)-x(t)|}{s^{\lambda}}=\infty, \\
& \lim _{s \downarrow 0} \sup \frac{|x(t-s)-x(t)|}{s^{\lambda}}=\infty
\end{aligned}
$$

for almost every $t \in T$ relative to the Lebesgue measure.

The proof of this theorem is based on a lemma which we prove in $\S 3$. The theorem itself is proved in $\$ 4$.

3. Lemma. Let $\lambda>\alpha / 2$. Then for every $t \in(0,1)$ there exists a subset of $C, \Gamma_{t} \in \mathcal{C}^{*}$ with $m_{G}\left(\Gamma_{t}\right)=1$ such that every $x \in \Gamma_{t}$ satisfies (5) and (6) at $t$.

Proof. Let $h>0, s>0$ and 


$$
\Gamma_{t, h, a}=\left\{x \in C ;|x(t+s)-x(t)| \leqq h s^{\lambda}\right\} .
$$

Then $\Gamma_{t, h, s} \in F_{C}$ and, by (4) and the fact that $\{\xi(t), t \in T\}$ is a Gaussian process, we have

$$
m_{G}\left(\Gamma_{t, h, s}\right)=\frac{1}{(2 \pi)^{1 / 2} \sigma_{t, t+8}} \int_{|\eta| \leq h 8^{\lambda}} \exp \left\{-\frac{\eta^{2}}{2 \sigma_{t, t+\varepsilon}^{2}}\right\} d \eta
$$

where

$$
\stackrel{2}{\sigma_{t, t+8}}=E\left\{|\xi(t+s)-\xi(t)|^{2}\right\} \geqq a s^{\alpha}
$$

according to (3). Thus

$$
m_{G}\left(\Gamma_{t, h, s}\right) \leqq(2 / \pi a)^{1 / 2} h s^{\lambda-\alpha / 2} .
$$

Since $\lambda-\alpha / 2>0, \lim _{s \downarrow 0} m_{G}\left(\Gamma_{t, h, s}\right)=0$. Let $\left\{s_{j}\right\}$ be a sequence of positive numbers such that $\sum_{j=1}^{\infty} s_{j}^{\lambda-\alpha / 2}<\infty$ and consequently $\sum_{j=1}^{\infty} m_{G}\left(\Gamma_{t, h, s_{j}}\right)<\infty$. By the Borel-Cantelli Theorem, $m_{G}\left(\Gamma_{t, h}\right)=1$ where $\Gamma_{t, h}=\lim _{j \rightarrow \infty}$ inf $\Gamma_{t, h, s_{j}}^{c}$. Thus, from the definition of $\Gamma_{t, h, s}$,

$$
\lim _{s \downarrow 0} \sup \frac{|x(t+s)-x(t)|}{s^{\lambda}} \geqq h \quad \text { for } x \in \Gamma_{t, h} \text {. }
$$

Let $\Gamma_{t}^{+}=\bigcap_{h=1}^{\infty} \Gamma_{t, h}$. Then $m_{G}\left(\Gamma_{t}^{+}\right)=1$ and

$$
\lim _{s \downarrow 0} \sup \frac{|x(t+s)-x(t)|}{s^{\lambda}}=\infty \quad \text { for } x \in \Gamma_{t}^{+} .
$$

Similarly, there exists a subset $\Gamma_{\bar{t}}$ of $C$ with $m_{G}\left(\Gamma_{\bar{t}}\right)=1$ such that

$$
\lim _{s \downarrow 0} \sup \frac{|x(t-s)-x(t)|}{s^{\lambda}}=\infty .
$$

We only have to take $\Gamma_{t}=\Gamma_{t}^{+} \cap \Gamma_{t}^{-}$to complete the proof of the lemma.

4. Proof of the Theorem. Consider the product measure $m=m_{G}$ $\times m_{L}$ on the product space $C \times T$, where $m_{L}$ is the Lebesgue measure on $T$. Let $\Gamma$ be the subset of $C \times T$ consisting of those elements $(x, t)$ for which (5) and (6) hold. We show that $\Gamma$ is a measurable subset of $C \times T$ by showing that the two functions of $(x, t), \lim \sup _{s}+0 s^{-\lambda} \mid x(t+s)$ $-x(t) \mid$ and $\lim \sup _{s+0} s^{-\lambda}|x(t-s)-x(t)|$, are measurable. We assume that each $x \in C$ has been extended beyond $T$ to be constant so that $x(t)$ is continuous in an interval containing $T$ in its interior. Now

$$
\lim _{s \downarrow 0} \sup \frac{|x(t+s)-x(t)|}{s^{\lambda}}=\lim _{\boldsymbol{t} \rightarrow \infty}\left\{\sup _{0<s<1 / \mathbf{k}} \frac{|x(t+s)-x(t)|}{s^{\lambda}}\right\} .
$$


Let $\left\{s_{j}\right\}$ be a countable dense subset of $(0,1 / k)$; so that from the continuity of $s^{-\lambda}|x(t+s)-x(t)|$ as a function of $s$ in $(0,1 / k)$, we have

$$
\sup _{0<s<1 / \mathbf{k}} \frac{|x(t+s)-x(t)|}{s^{\lambda}}=\sup _{j} \frac{\left|x\left(t+s_{j}\right)-x(t)\right|}{s_{i}^{\lambda}} .
$$

Since each $s_{j}^{-\lambda}\left|x\left(t+s_{j}\right)-x(t)\right|$ is a measurable function of $(x, t)$ on $C \times T$ so is $\lim \sup _{s \downarrow} s^{-\lambda}|x(t+s)-x(t)|$. Similarly,

$$
\lim _{s \downarrow 0} \sup s^{-\lambda}|x(t-s)-x(t)|
$$

is measurable, and $\Gamma$ is measurable.

Now for each $t \in T$, let

$$
\Gamma(t)=\{x \in C ;(x, t) \in \Gamma\} .
$$

For almost every $t, \Gamma(t)$ is a measurable subset of $C$ and for every $t$, $\Gamma(t) \supset \Gamma_{t}$ of the Lemma. Since $m_{G}\left(\Gamma_{t}\right)=1=m_{G}(C)$, we have

$$
\int_{0}^{1} m_{G}(\Gamma(t)) m_{L}(d t)=\int_{0}^{1} m_{G}\left(\Gamma_{t}\right) m_{L}(d t)=1
$$

and hence by Fubini's theorem, $m(\Gamma)=1$ and, furthermore,

$$
0=m(C \times T-\Gamma)=\int_{c} m_{L}\{t \in T ;(x, t) \notin \Gamma\} m_{G}(d x) .
$$

Thus, for almost every $x \in C$,

$$
m_{I}\{t \in T ;(x, t) \notin \Gamma\}=0 ;
$$

that is, for almost every $x \in C$,

$$
m_{L}\{t \in T ;(x, t) \in \Gamma\}=1 .
$$

This completes the proof of the Theorem.

\section{BIBLIOGRAPHY}

1. J. L. Doob, Stochastic processes depending on a continuous parameter, Trans. Amer. Math. Soc. 42 (1937), 107-140.

2. A. N. Kolmogoroff, Grundbegriffe der Wahrscheinlichkeitsrechnung, Ergebnisse der Mathematik, No. 3, (1933).

3. M. Loève, Probability theory, Princeton Univ. Press, Princeton, N. J., 1963.

4. P. T. Strait, Sample function regularity for Gaussian processes with the parameter in a Hilbert space, Ph.D. Thesis, New York Univ., 1965.

5. N. Wiener, Differential space, J. Math. and Phys. 2 (1923), 131-174.

6. - Generalized harmonic analysis, Acta Math. 55 (1930), 117-258.

University of California, Irvine 